ROCZNIKI HUMANISTYCZNE

Tom LXIX, zeszyt $8-2021$

DOI: https://doi.org/10.18290/rh21698-9

\title{
COLLOCATIONS VERBO-NOMINALES AVEC GNIEW (COLÈRE) DANS BRAT NASZEGO BOGA (KAROL WOJTYŁA) ET LEUR TRADUCTION EN FRANÇAIS ET EN NÉERLANDAIS
}

\author{
VERBO-NOMINAL COLLOCATIONS WITH GNIEW (ANGER) \\ IN BRAT NASZEGO BOGA (KAROL WOJTYŁA) \\ AND THEIR TRANSLATION INTO FRENCH AND DUTCH
}

\begin{abstract}
This article reports on an analysis of the translation of collocations with the noun gniew (anger) in Karol Wojtyła's play Brat Naszego Boga ('Brother of our God') into French, and proposes translations of these collocations into Dutch. For the semantic-discursive analysis of collocations with gniew (anger), we established a methodological model in which we integrated based on Eckman's definition of anger - elements of this emotion (as a process) and their linguistic realisation, which takes the form of a verb-noun collocation (of the $\mathrm{V}+\mathrm{N}$ or $\mathrm{N}+\mathrm{V}$ type) according to the cognitive approach of collocations proposed by Śliwa.

The study opens up a contrastive perspective in order to identify possible difficulties in the translation of verb-nominal collocations with gniew (anger). In order to facilitate a more detailed description of the degree of equivalence in the translation of these collocations, we have resorted to Camugli Gallardo's scheme.

This analysis allows us to state that a semantic-discursive approach applied to the comparative analysis of the translation of certain lexical units - such as collocations - could be adopted as a starting point for defining the criteria for evaluating the quality of a translation.
\end{abstract}

Key words: nouns denoting emotion; anger; ontological structure; verb-noun collocations; translation strategies; equivalence.

Dr hab. Muriel WATERLOT - HDR en linguistique à la Chaire de linguistique néerlandaise de 1'Université Catholique Jean Paul II de Lublin; adresse postale: Katedra Języka Niderlandzkiego, Katolicki Uniwersytet Lubelski Jana Pawła II, Al. Racławickie 14, 20-950 Lublin, Pologne; e-mail: murielwaterlot@kul.pl; ORCID: https://orcid.org/0000-0002-1665-2543. 


\section{INTRODUCTION}

Toutes les langues naturelles possèdent des mots et des expressions qui aiment être à proximité d'autres mots et expressions ; elles possèdent également des mots et des expressions qui évitent « la compagnie »d'autres éléments lexicaux. Ce phénomène est souvent désigné par le terme de collocation.

Cette recherche a pour objectif premier l'élaboration d'un cadre méthodologique pour une analyse sémantico-discursive des collocations (et leur traduction) exprimant l'une des six émotions de base (Ekman), notamment la colère $^{1}$. En prenant le polonais comme langue de départ nous analysons, en utilisant cette méthodologie, la traduction de collocations avec le nom gniew (colère) dans la pièce de théâtre de Karol Wojtyła Brat naszego Boga (Frère de notre Dieu) écrite dans les années 1944-1950 et traduite en français par Zofia Bobowicz \& Jean Offredo (1982).

En un premier temps, nous présenterons succinctement le contenu de cette pièce et nous préciserons l'importance et le rôle de la colère. Après avoir expliqué un certain nombre de concepts clés pertinents pour cette analyse, nous décrirons l'approche méthodologique («modèle intégré ») défini à partir d'un modèle réduit du système d'émotion basique de Matsumoto \& Ekman dans lequel ont été intégrées des descriptions sémantiques de collocations avec le nom colère correspondant aux trois étapes du processus émotionnel lié à cette émotion: stimulus - réaction - comportement.

Nous adopterons une perspective contrastive pour repérer d'éventuelles difficultés/restrictions que pose la traduction d'un certain nombre de collocations verbo-nominales avec gniew du polonais vers le français (colère) et le néerlandais $\left(\right.$ woede ${ }^{2}$ ). Nous proposons d'étudier ici le degré de correspondance (syntaxique [composition] et sémantique [signification]) de la traduction de ces collocations (du type nom + verbe et verbe + nom), et les stratégies de traduction (niveau d'équivalence) adoptées par le traducteur dans le texte français. Cette analyse nous permettra de proposer des traductions de ces unités lexicales en néerlandais en vue de la future traduction néerlandaise de la pièce Brat naszego Boga («Frère de Notre Dieu »).

\footnotetext{
${ }^{1}$ Plusieurs études sur les collocations avec des noms de sentiments ont été realisées par Anna Krzyżanowska (voir par exemple Krzyżanowska, « Sur la place », « Traduire les émotions »).

${ }^{2}$ Le mot colère peut egalement être traduit en néerlandais par toorn. Selon le dictionnaire Van Dale Groot woordenboek der Nederlandse taal, toorn est un mot quelque peu archaïque pour désigner la colère. Dans les versions plus récentes de ce même dictionnaire (par exemple celle de 2005), la signification du mot toorn est illustrée par des phrases et proverbes tirés de la Bible. En effet, dans le contexte de Dieu par exemple, le mot toorn semble plus commun en néerlandais que woede.
} 


\section{LA PIÈCE BRAT NASZEGO BOGA ET L'IMPORTANCE DE LA COLÈRE}

La pièce de théâtre Brat naszego Boga a été écrite par Karol Wojtyła après la Seconde Guerre, dans les années 1944-1950, pendant les années d'occupation nazie et de stalinisme. Wojtyła a écrit cette pièce alors qu'il était étudiant et ouvrier, et ensuite vicaire à l'église Saint-Florian de Cracovie. En tant que pape Jean-Paul II, il a confirmé à plusieurs reprises sa fascination pour la personne et la vie d'Adam Chmielowski avec qui il partageait des expériences de vie semblables et les mêmes valeurs (Olszewska).

La structure de ce drame écrit par Wojtyła se déroule à trois niveaux : le niveau externe (réaliste), interne (psychologique) et celui de la didascalie.

L'acte I «Pracownia przeznaczeń » (« l'Atelier des destins ») se déroule dans l'atelier d'Adam Chmielowski. Parmi les discussions sur la mission de l'art, son autonomie et son rôle dans la société, il y est également question de la vocation et l'attitude de l'homme envers la société. Ceci entraîne un échange de vues sur le devoir envers la misère humaine qui se résume à la question suivante : comment être responsable d'un citoyen qui a gâché sa vie et se trouve maintenant au fond du gouffre ? L'évènement clé qui marque ce changement se déroule à la fin de l'hiver, lorsque - en cherchant à s'abriter de la pluie - Adam trouve refuge dans un refuge pour sans-abri. Depuis lors, Adam ne peut s'empêcher de penser au problème de la pauvreté.

Ces discussions sont complétées par l'acte dans lequel se déroule le dialogue avec un étranger qui représente l'être profond du protagoniste. Leur conversation porte sur la colère naissante des gens et celle éprouvée par le protagoniste Adam qui rejette la tentation de transformer sa colère en une œuvre d'art (Olszewska 134).

Le motif de la colère revient dans l'acte II « W podziemiach gniewu » ( « Les sous-sols de la colère »). Adam se rend au foyer municipal, mais il est traité de façon hostile par les mendiants qui ne lui font pas confiance car il est le représentant des gens riches et heureux qui ne savent pas ce que signifie être au fond de la misère. Adam découvre que sa place est parmi ces mendiants et non pas dans les ateliers de peinture. La puissance de la miséricorde de Dieu est révélée à Adam dans son tableau Ecce Homo où il peint le visage du Christ portant une couronne d'épines en qui il reconnait le visage des pauvres. Dans une des scènes du drame, Adam rencontre " l'Autre » qui déclare qu'Adam est naïf de croire que la miséricorde changera le sort des sans-abris. "L'Autre » encourage les mendiants à la révolte car, selon lui, seule la force peut changer les lois de ce monde. Il voit la possibilité de ce 
changement dans une révolution sociale fondée sur l'idée de la lutte des classes. Il essaie donc de persuader les mendiants de se révolter contre les riches. Adam comprend que la pauvreté peut les pousser à la révolte car ils ont le droit moral de le faire. Cependant, son attitude est différente car il veut " éduquer la colère pour qu'elle mûrisse comme force créatrice » (Karol Wojtyła francais [suivant : KW-FR] 347) et non pas à « l'exploiter en l'utilisant comme un simple outil » (KW-FR 347) pour déclencher une révolution sociale. Nous voyons ici l'opposition entre la colère qui a une dimension spirituelle d'une part (impliquant la transformation spirituelle des pauvres rendue possible grâce à la miséricorde) et celle qui a une dimension sociale d'autre part (Olszewska 136).

L'acte III, intitulé « Dzień brata » (« Une journée dans la vie du frère »), se déroule plusieurs années plus tard dans un foyer pour les pauvres qui a été transformé en couvent. Adam est devenu frère Albert et il explique à ses confrères que la pauvreté est liée à la souffrance, mais qu'ils doivent l'accepter comme un don. Il leur apprend qu'en s'approchant du Christ, la croix unie à Sa Croix transforme la chute de 1'homme en bien, et son esclavage en liberté (Olszewska 137). Ceci le conduit à la fin du drame - lorsqu'il apprend que des émeutes, alimentées par une "grande et juste colère », avaient éclaté dans la ville - de prononcer des paroles significatives : "Mais ce dont je suis sûr, c'est que moi, j'ai choisi une plus grande liberté » (KW-FR 373). Pour le frère Albert il s'agit de la liberté de toute forme d'agression, de la violence, de viol. Elle consiste en la liberté de vivre et de faire Miséricorde (Olszewska 137). Sous l'emprise de la Miséricorde, la colère peut devenir une force créatrice qui aboutit à une transformation spirituelle de l'être humain.

\section{DÉFINITIONS: CONCEPT, COLÈRE, COLLOCATION}

Avant de commencer notre analyse, nous nous attarderons sur un certain nombre de définitions qui sont importantes pour comprendre le cadre théorique. Tout d'abord, nous clarifierons ce que nous entendons par le terme concept, que nous relierons à une définition du mot émotion. Pour rendre ce lien possible, nous allons faire le lien entre les collocations et la représentation conceptuelle de l'émotion dont nous pouvons d'une part faire l'expérience et ensuite décrire/verbaliser les propriétés e.a. du réel, et que d'autre part nous pouvons considérer comme un processus dynamique dans lequel différents facteurs (personnels ou relationnels) peuvent exercer une influence et/ou jouer un rôle. 


\subsection{DEFINITION DU CONCEPT ET DE LA STRUCTURE CONCEPTUELLE}

Avant d'entamer notre analyse, nous aborderons en quelques mots la problématique de la signification du terme concept, et comment les émotions (ici : la colère) peuvent être décrites à l'aide de concepts.

Pour une étude orientée vers des concepts (comme la nôtre), nous pouvons adopter une approche onomasiologique qui a pour point de départ les concepts pour aboutir à la dénomination (Śliwa, "Parlons cerises » 414). Les concepts ont donc besoin d'exister dans l'esprit du sujet parlant et d'être mis en relation avec un référent qui est un objet de la réalité (cf. Cabré 6). Compte tenu de l'importance de la participation du locuteur et de sa connaissance du monde, nous aboutissons chez Gilson (1969: 142-143, cité par Śliwa, «Parlons cerises » 416) qui définit le concept comme un « acte particulier de l'intellect » et comme le "résultat de la pensée ». Cette position théorique basée sur la métaphysique réaliste nous permet de considérer la construction de la structure ontologique (de nature conceptuelle) en " tenant compte de la structure ontique et des liens logiques cause-effet » (Śliwa, «Parlons cerises » 416). En d'autres termes, cela nous permet « de relier le réel avec le conceptuel en vertu duquel les propriétés du réel transcendent dans l'intellect sous forme de concepts » (ibidem).

Dans notre analyse, il s'agit de rapporter les propriétés du réel de l'émotion 'colère' (processus ou état affectif) qui par l'activité intellectuelle/cognitive se transforment spontanément en concepts reliés entre eux par des relations logiques.

Pour de nombreux traducteurs professionnels, l'un des problèmes centraux qu'ils rencontrent est la recherche d'une équivalence conceptuelle et terminologique entre le texte source et le texte cible. En adoptant une approche métaphysique réaliste, le traducteur pourra avoir recours aux propriétés du réel à partir de leur représentation conceptuelle commune aux usagers de la langue source et la langue cible, et rendre ainsi consciente et explicite l'étape de la déverbalisation grâce aux schémas conceptuels basés sur les structures ontologiques.

\subsection{DEFINITION DE L’EMOTION 'COLERE’}

Puisqu'aucun dictionnaire ne peut prétendre à lui seul à l'exhaustivité terminologique, il nous faudra tout d'abord trouver une base pour identifier l'organisation conceptuelle globale de certaines collocations liées au concept 'colère' car l'interaction entre cognition et dénomination est essentielle pour 
déterminer la signification exacte des termes et pour trouver les justes équivalents dans la langue cible. Examinons d'abord l'aspect cognitif et noncognitif du concept 'émotion'.

Il existe de nombreuses définitions du terme émotion. Dans notre analyse, nous considérons l'émotion comme « un ensemble de variations épisodiques dans plusieurs composantes de l'organisme en réponse à des événements évalués comme importants pour l'organisme » (Scherer dans Coppin \& Sander 13). Selon cette définition, l'émotion est constituée de cinq composantes : l'évaluation cognitive, les changements psychophysiologiques, l'expression motrice, les tendances à l'action et le sentiment subjectif.

Nous pouvons constater que le concept 'émotion' fait l'objet d'un débat intense dans la littérature, avec comme deux extrêmes : la théorie non-cognitive (approche physiologique de James) d'une part et la théorie cognitive (approche intellectualiste de Solomon et de Nussbaum) d'autre part. Dans notre analyse, nous opterons pour une position dans laquelle les symptômes physiques et le jugement (cognitif) sont tous deux importants.

Afin de relier le réel avec le conceptuel (ou de définir le concept 'colère'), et en vue de réunir les propriétés inhérentes de la colère, notre attention ira vers la séquence définitionnelle du type analytique (logique). Ces composantes (qui décrivent les propriétés inhérentes de la colère) nous fourniront un point de départ pour établir une base/un cadre ontologique pour étudier les collocations avec les noms de ce concept. Pour comprendre la structure ontologique du concept 'colère', nous avons recours au savoir des spécialistes en matière de la psychologie qui mettent en avant le déclenchement et le déroulement (manifestations somatiques) de ce processus émotionnel.

Stoessel (158) met en avant le rôle de l'activation physiologique dans le déclenchement et le déroulement du processus émotionnel, le stimulus étant une réaction liée à une blessure physique ou mentale, un manque, une frustration entraînant un déclenchement de manifestations physiques (symptômes) ou psychologiques (réactions) de la part de la personne concernée, celles-ci pouvant cependant être contenues, voire dissimulées.

Cette définition nous révèle trois étapes dans le processus émotionnel lié à la colère : stimulus - réaction - comportement que nous retrouvons également dans la représentation du système d'émotion basique de Matsumoto et Ekman: 
Le système d'émotion basique

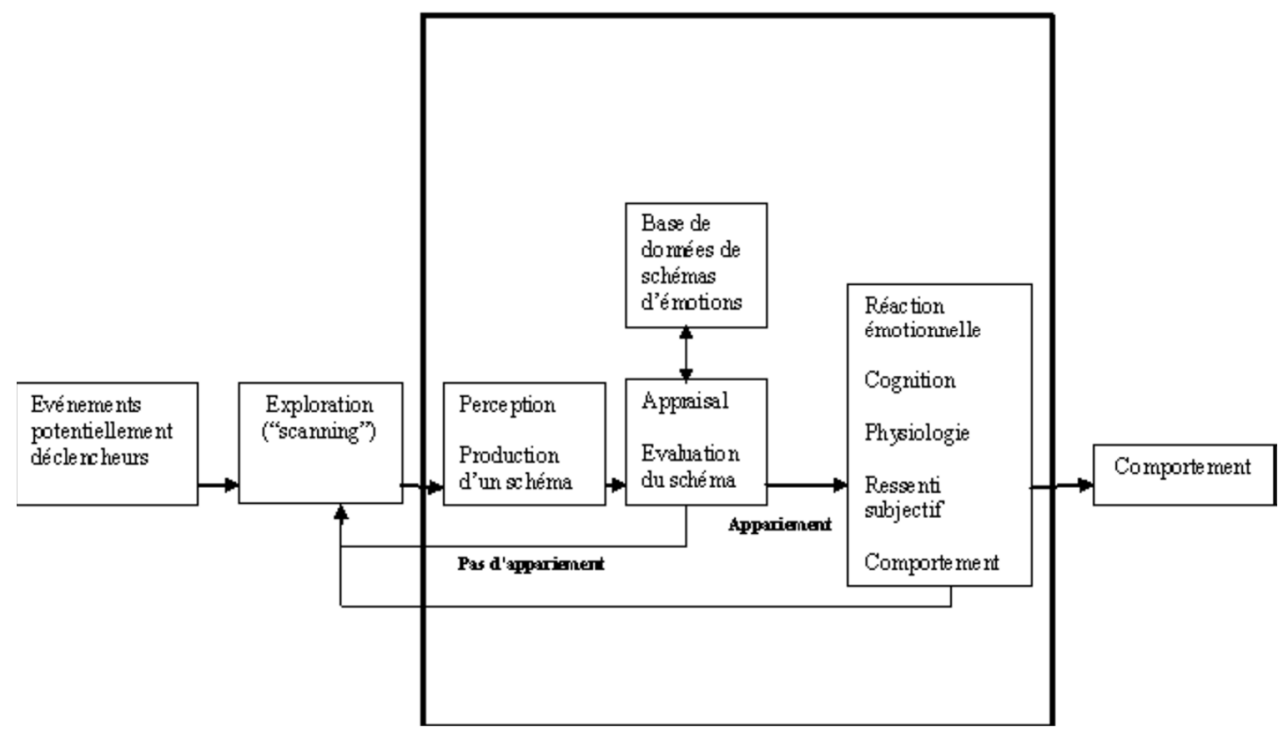

Schéma 1. Représentation du système d'émotion basique (Matsumoto \& Ekman)

Lorsque nous considérons l'émotion selon cette représentation, elle est constituée de trois composantes de base : les événements déclencheurs (cf. 'stimulus' ou 'déclenchement'), les réactions émotionnelles (le développement au niveau cognitif, subjectif et physiologique) et l'expression de l'émotion ou les tendances à l'action (comportement/extinction). Ceci nous amène à la représentation simplifiée du système d'émotion basique de Matsumoto et Ekman (cf. schéma 2) :

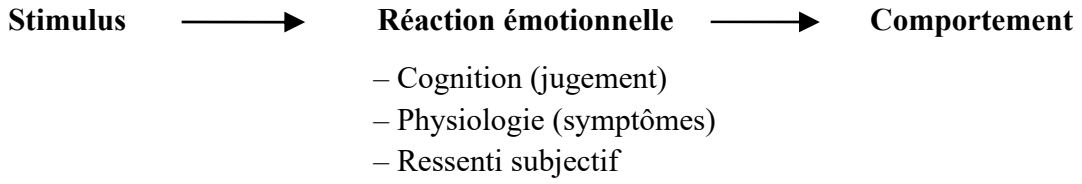

Schéma 2. Représentation simplifiée du système d'émotion basique (Matsumoto \& Ekman)

Comme nous le verrons au paragraphe 3 en décrivant la méthodologie de notre analyse, ce schéma nous servira de base pour relier l'expérience de l'émotion 'colère' par le sujet parlant (et les entités du réel qui apparaissent dans la pièce théâtre Brat naszego Boga) - à travers sa conceptualisation par 
son intellect qui évalue le réel - et son expression (dans une des trois phases de l'émotion) sous forme de collocations verbo-nominales spécifiant une propriété inhérente ou relationnelle de la colère (analyse sémantico-discursive).

\subsection{UNE APPROCHE COGNITIVE DE LA COLLOCATION}

Parmi les différentes conceptions de la collocation ${ }^{3}$, nous retenons celle qui permet de réaliser les principes méthodologiques de la description de la colère évoquées ci-dessus. Nous retenons notamment la conception 'étroite' de la collocation, à savoir : le caractère binaire selon lequel la collocation est composée de deux mots ou de deux lexies et la dissymétrie des composants de la collocation. Parmi les paires de parties du discours (des constituants spécifiques) que les collocations peuvent constituer, Hausmann (1010) énumère : substantif + adjectif (épithète) ; substantif + verbe ; verbe + substantif (objet) ; verbe + adverbe ; adjectif + adverbe ; substantif + (prép. $)+$ substantif.

Pour les besoins de notre analyse des collocations en discours, nous nous appuierons sur trois dimensions des collocations, définies par Lerat (102). Ce dernier en effet distingue trois niveaux des collocations qui permet trois niveaux d'analyse de collocations suivant l'approche pragmatique, syntaxique et sémantique, commentés par Śliwa (« Coeur et sentiments»91-92) :

1) le niveau «pragmatique » (Śliwa, "Coeur et sentiments » 91) que nous pouvons appeler le niveau discursif (exprimé par les verbes en contexte); cette dimensions nous permet de repérer une collocation dans un texte ou un discours (ici: passages d'une pièce de théâtre), avec toutes les marques énonciatives (surtout les collocations verbo-nominales où le verbe a une forme flexionnelle et une modalité) ;

2) le niveau « sémantique » (qui exprime le sens) où Lerat introduit le niveau conceptuel (= concept et les composantes conceptuelles) lié au sens de combinaison de lexème préféré et qui «s'intéresse à la connectabilité conceptuelle » (Lerat, cité par Śliwa, « Coeur et sentiments » 91);

3) le niveau « syntaxique » qui est " lié à l'aspect syntaxique de combinaison de lexème préféré, expose la combinaison syntagmatique » (ibidem) selon lequel nous pouvons schématiser les types (constructions syntaxiques) des collocations $(\mathrm{N}+\mathrm{Adj}, \mathrm{V}+\mathrm{N} / \mathrm{N}+\mathrm{V}$, etc.). En ce qui concerne ce niveau, seules les collocations verbo-nominales seront prises en compte dans notre analyse.

\footnotetext{
${ }^{3}$ Les premières études sur ce que nous appelons aujourd'hui des collocations, apparaissent au début du $20^{\mathrm{ème}}$ siècle avec le Traité de stylistique française (1909) de Bally. Les différentes conceptions des collocations ont été présentées par Tutin \& Grossmann. Parmi les partisants de la conception 'étroite' de la collocation, nous retrouvons Haussman, Cruse et Mel'cuk.
} 
Le schéma courant de la structure binaire de la collocation est le suivant:

$$
\text { Mot de base }+ \text { collocatif }=\text { collocation }
$$

Suivant la démarche onomasiologique adoptée, nous proposons de commencer par le niveau référentiel/conceptuel (c'est-à-dire la conceptualisation de la réalité de l'émotion). Lorsque nous introduisons la dimension référentielle/conceptuelle dans l'analyse sémantico-syntaxique des collocations (cf. Śliwa, "Une approche cognitive » 292), nous pouvons supposer que dans le schéma ci-dessus, le mot de base, qui est le nom $(\mathrm{N})$, réfère à une catégorie du réel/au concept (ici : la colère' qui est 'responsable' de la sélection du collocatif (spécifiant une propriété de la colère).

$\mathrm{Au}$ niveau conceptuel, la structure fondamentale bipartite de la collocation est composée du spécifié (l'entité conceptuelle superordonnée) et du spécifiant qu'est la propriété perçue qui peut être :

- inhérente (dimensions), exprimée dans les collocations nominaux-adjectivales, par exemple: $(\mathrm{Adj}+\mathrm{N})$ wielki gniew (grande colère) où la propriété spécifiante est exprimée par l'adjectif;

- inhérente (dynamique), exprimée dans les collocations $(\mathrm{N}+\mathrm{V})$ gniew rośnie (la colère monte) où la propriété spécifiante est exprimée par le verbe;

- relationnelle, exprimée dans les collocations verbo-nominales, par exemple: $(\mathrm{V}+\mathrm{N})$ wychować gniew (éduquer la colère) où la propriété est exprimée par le verbe collocatif (d'après Śliwa, "Testament »141).

Comme nous le verrons dans le paragraphe suivant, ces collocations verbonominales avec le $\mathrm{N}$ colère seront extraites du discours, c'est-à-dire des passages de la pièce de théâtre en polonais pour finalement être comparées à leur traduction en langue française et néerlandaise.

\section{CADRE MÉTHODOLOGIQUE: MODÈLE INTÉGRÉ POUR UNE ANALYSE SÉMANTICO-DISCURSIVE DES COLLOCATIONS AVEC COLÈRE}

Pour l'analyse sémantico-discursive des collocations avec gniew (colère), nous avons établi un modèle méthodologique dans lequel nous avons intégré par le biais de la définition de l'émotion 'colère' (cf. 2.2.) - des éléments d'une processus de cette émotion et leur réalisation linguistique qui se présente sous la forme d'une collocation verbo-nominale du type $(\mathrm{V}+\mathrm{N}$ ou $\mathrm{N}+\mathrm{V})$. 
Dans ce paragraphe, nous présentons (à base de la définition de la colère présentée au paragraphe 2.1.) ce modèle intégré, fondé sur la représentation du système d'émotion basique de Matsumoto et Ekman (cf. Schéma 1 au paragraphe 2.2.) dans lequel, par le biais d'une approche cognitive, nous avons intégré - pour chacune des phases qui décrivent le processus de l'émotion 'colère' - le sens d'un collocatif qui se rapporte à une des trois phases de son déroulement et à la manière dont elle est expérimentée (par un individu ou par un groupe de personnes) dans sa phase initiale (déclenchement), dans son déroulement (développement) et dans sa phase finale (extinction).

La structure actantielle des verbes collocatifs (désignant les propriétés inhérentes dynamiques et les propriétés relationnelles) sera reliée au schéma basique de l'émotion où les actants désignent respectivement le stimulus, l'expérienceur et un participant. Ainsi, dans la pièce de théâtre Brat naszego Boga par exemple, des personnages qui portent des noms tels que Mówca (« l'Homme ») ou Nieznajomy («l'Inconnu », le moi profond du protagoniste Adam et futur frère Albert) stimulent la colère des mendiants.

Dans le schéma ci-dessous, nous voyons qu'à chaque phase du processus de l'émotion, il y a deux agents qui peuvent exercer une influence sur l'émotion (c'est-à-dire sur sa phase de début et de fin).

\begin{tabular}{|c|c|c|}
\hline I. Stimulus (causatif) & $\begin{array}{l}\text { II. Réaction émotionnelle de } \\
\text { l'expérienceur } \\
\text { (développement/déroulement) }\end{array}$ & $\begin{array}{l}\text { III. Comportement de } \\
\text { l'expérienceur } \\
\text { (conséquences/extinction) }\end{array}$ \\
\hline $\begin{array}{l}\text { Collocatifs exprimant le } \\
\text { déclenchement de l'état } \\
\text { émotionnel (stimulus) }\end{array}$ & $\begin{array}{l}\text { Collocatifs exprimant le début ou le } \\
\text { déroulement de l'état émotionnel } \\
\text { Cognition (jugement, opinion, } \\
\text { connaissance) } \\
\text { Collocatifs exprimant l'expérience } \\
\text { d'une attitude cognitive lors de } \\
\text { l'expérience de l'émotion }\end{array}$ & $\begin{array}{l}\text { Collocatifs exprimant l'intensité } \\
\text { de l'état émotionnel et } \\
\text { collocations exprimant la fin de } \\
\text { l'état émotionnel } \\
\text { Participants : }\end{array}$ \\
\hline \multirow{2}{*}{$\begin{array}{l}\text { ou } \\
\text { b. Agent externe } \\
\text { (individus, groupes, } \\
\text { personnes, évènements, } \\
\text { obstacles ...) }\end{array}$} & $\begin{array}{l}\text { Physiologie (symptômes) } \\
\text { Collocatifs exprimant le } \\
\text { déroulement et la manifestation } \\
\text { physiologique de l'émotion }\end{array}$ & \multirow{2}{*}{$\begin{array}{l}\text { Participants : } \\
\text { a. L'expérienceur } \\
\text { ou } \\
\text { b.Agent externe (individus, } \\
\text { groupes, personnes, évènements, } \\
\text { obstacles ...) }\end{array}$} \\
\hline & $\begin{array}{l}\text { Ressenti subjectif } \\
\text { Collocatifs exprimant les } \\
\text { réactions, les comportements } \\
\text { personnels de l'individu lors de } \\
\text { l'expérience de l'émotion }\end{array}$ & \\
\hline
\end{tabular}

Schéma 3. Modèle intégré d'analyse sémantico-discursive des collocations avec une émotion 
Outre les critères qui concernent les 'phases' de la colère (cf. : a) déclenchement, b) développement, c) extinction), nous avons introduit ceux qui concernent l'agent externe ou le causeur de la colère dans la phase du déclenchement, ainsi que dans la phase d'extinction - la colère pouvant résulter en une sorte de 'manipulation' ou de 'modélisation' de la colère.

Dans le cas d'une collocation verbo-nominale $(\mathrm{V}+\mathrm{N} / \mathrm{N}+\mathrm{V})$, elle fera référence - par le biais des fonctions intellectuelles/cognitives - soit à la dynamique (un processus) de la colère expérimentée par une personne (dimension personnelle) ou plusieurs personnes (dimension collective), soit à une propriété qui peut être relationnelle (dans le cas où par exemple une action/émotion est provoquée par un agent extérieur (une personne ou un groupe de personnes, un événement ou une situation) auprès d'un expérienceur (d'une ou plusieurs personnes) ou utilisée/manipulée comme un instrument pour atteindre un objectif (ici : social ou spirituel) (d'après Śliwa, « Une approche cognitive » 292).

Comme nous pouvons le voir sur le schéma 3, dans chacune des trois phases du système d'émotion il y des participants. Dans la phase du déclenchement et de l'extinction de la colère nous avons d'une part l'expérienceur dans lequel est localisée la colère - et d'autre part des agents externes ( $=$ les stimulus: individus, groupes, personnes, évènements, obstacles ...) qui agissent sur l'expérienceur. Dans la phase du déroulement, l'expérienceur occupe une position centrale en tant que celui qui en fait expérience.

Dans la pièce de théâtre Brat naszego Boga, l'expérienceur de la colère sont d'une part le protagoniste Adam (le futur Frère Albert), et d'autre part les mendiants/les pauvres (= groupe de personnes).

Les agents externes qui peuvent être des stimulus sont principalement des personnes (cf. Mówca - « l'Homme », Nieznajomy - «l'Inconnu ») et des évènements (cf. le contexte situationnel d'injustice, de pauvreté et d'impuissance qui suscite la colère des protagonistes).

\section{2. ÉQUIVALENCE ET DEGRES D’EQUIVALENCE DANS LA TRADUCTION}

Le fait que les collocations soient des unités préfabriquées, forgées par l'usage, à caractère essentiellement idiosyncrasique peut rendre problématique la traduction de ces combinaisons lexicales. D'une langue à l'autre, les bases ne sélectionnent pas nécessairement les mêmes collocatifs - par exemple : Fr. une peur bleue - P1 : wielki strach. Cet exemple illustre qu'il peut y avoir un manque de correspondance dans le transfert d'une langue à une autre, une impossibilité de traduire une collocation mot à mot. Le traducteur devra donc maîtriser les systèmes collocationnels des langues impliquées dans la traduction. 
En l'absence de cette maîtrise, les collocations constituent de véritables difficultés dans la traduction.

Afin de détecter des stratégies adoptées par le traducteur dans la traduction des collocations verbo-nominales avec les noms de 'colère' du polonais vers le français (et le néerlandais), nous analyserons la traduction du corpus de collocations avec les noms de 'colère' recueillies dans la traduction française de Brat naszego Boga en fonction de leur degré d'équivalence. À cette fin, nous aurons recours au schéma de Camugli Gallardo (dans González Rey 234) qui distingue cinq types d'équivalences:

a) les expressions rigoureusement équivalentes tant du point de vue lexical, syntaxique que sémantique ;

b) les équivalences qui présentent la même signification portée par une structure analogue mais avec une légère variation lexicale ;

c) les équivalences partageant la même aire lexico-sémantique mais avec des structures syntaxiques différentes;

d) les équivalences totalement différentes tant du point de vue lexical, syntaxique que sémantique ;

e) les équivalences «fausses », deux expressions pouvant présenter des structures et un lexique proches mais dont les acceptions sont tout à fait divergentes.

Nous avons choisi ce modèle parce qu'il nous permet de donner un retour d'information sur trois niveaux : sémantique, le syntaxique et le lexical.

\section{ANALYSE DES COLLOCATIONS VERBO-NOMINALES AVEC GNIEW (COLĖRE) ET LEURS TRADUCTIONS EN FRANÇAIS ET EN NÉERLANDAIS}

Dans la suite de notre analyse, nous prendrons en compte les collocations dont le verbe collocatif exprime une phase de l'émotion (Tutin \& Grosmann 17). Nous allons analyser si, du point de vue de la traduction, nous pouvons constater des difficultés dans le choix du verbe collocatif en français (et en néerlandais). Ceci pourrait notamment être le cas lorsque le verbe est métaphorique.

Nous avons classifié les collocations verbo-nominales selon les trois phases du modèle intégré (cf. Schéma 3 ) qui réfèrent à une propriété perçue dans une des trois phases de l'émotion (I. Stimulus, II. Développement/Déroulement, II. Extinction/Conséquences). Ces propriétés étant de nature rela- 
tionnelle, seront analysées a) du point de vue de la manière dont un 'expérienceur' (une personne ou un groupe) éprouve l'émotion 'colère' et b) du point de vue de la manière dont la colère peut être suscitée ou utilisée par un 'agent externe' (= individu, groupe, personnes, évènements, obstacles).

\section{1. LES COLLOCATIFS EXPRIMANT LA PHASE INITIALE (STIMULUS - CAUSATIF)}

Comme nous l'avons vu au paragraphe 1, le déclenchement de la colère exprimé dans les collocations est principalement lié au contexte dans lequel se déroule la pièce, à savoir : un contexte situationnel d'injustice, de pauvreté et d'impuissance qui suscite la colère des protagonistes, ou les incitent à la provoquer et à la manipuler ou à la transformer parmi les mendiants à des fins précises (sociales ou spirituelles) auprès des personnes qui subissent ou se trouvent dans cette situation.

Sur la base de ce contexte, nous analyserons les collocatifs (Vinch) de la pièce exprimant la phase initiale ou le déclenchement de l'état émotionnel. Conformément à la psychologie humaine (à la réalité), ce déclenchement peut être éprouvé par un 'expérienceur' (une personne ou un groupe), ou causé/suscité par un 'agent externe' (= une personne/des personnes, des évènements). Dans ce dernier cas, les collocatifs peuvent donc également adopter une interprétation de causatif, introduisant un stimulus (agent externe) qui agit sur l'expérienceur.

Suivant cette logique, nous analyserons d'abord des exemples de collocations associées au déclenchement de la colère chez 'l'expérienceur', et ensuite celles provoquées par un 'agent externe'. Dans les deux cas, elles expriment une propriété de la colère située dans la dimension relationnelle.

\section{L'expérienceur:}

Dans l'exemple suivant, l'expérienceur de la colère est Adam (le futur Frère Albert):

- gniew wzbiera w kimś - se mettre en colère

- Idę dalej, bo czuję, że wzbiera we mnie gniew (KW-PL 70)

- (...) je finirai par me mettre sérieusement en colère (KW-FR 336)

Nous constatons dans ce premier exemple des équivalences différentes tant du point de vue sémantique, lexicale et syntaxique. Du point de vue sémantique, la différence se situe principalement dans l'expression de l'état initial: en polonais - l'intensité et augmentation de la colère et aspect duratif; le traducteur français utilise une collocation verbo-nominale qui exprime 
le commencement d'action ou d'état (aspect inchoatif): se mettre en colère. Nous sommes d'avis que le traducteur aurait pu se servir d'une traduction littérale 'je sens que la colère monte en moi' (qui réfère également, par un Vinch, au déclenchement de la colère ou de cet «état affectif passager, résultant du sentiment d'une agression, d'un désagrément, traduisant un vif mécontentement (...)»(L) afin de préserver l'image de l'homme qui, tel un vase vide, se remplit de colère.

Quant à la traduction néerlandaise de cette collocation, nous optons pour une traduction littérale avec un Vinch : de woede borrelt in iemand (mij) op .

\section{Agent externe:}

Dans la pièce Brat naszego Boga, nous constatons que dans la plupart des collocations, des personnages qui portent des noms tels que Mówca («l'Homme ») ou Nieznajomy («l'Inconnu'» ou le moi profond du protagoniste Adam et futur Frère Albert) sont les 'déclencheurs' de la colère des mendiants. Néanmoins, ils représentent en quelque sorte des 'déclencheurs indirects' à cette colère, qui est principalement suscitée par le contexte social d'injustice que nous devons considérer comme le 'déclencheur direct'. Par conséquent, nous traiterons les stimuli 'agent externe' représentant des personnes et des événements ensemble, dans une catégorie (cf. celle d' 'agent externe').

Dans l'exemple qui suit, nous voyons que l'agent externe (causatif) est une personne (cf. Mówca, «l'Homme ») qui provoque la colère nécessitant son déclenchement qui pourra entraîner un changement :

- potrzebować gniewu kogo - avoir besoin de la colère de quelqu'un

- Potrzeba tylko waszego gniewu (KW-PL 75)

- Nous n'avons besoin que de votre colère (KW-FR 342).

Les équivalences partageant la même aire lexico-sémantique mais avec des structures syntaxiques différentes : en polonais nous avons une expression impersonnelle (sans infinitif), tandis qu'en français nous avons un verbe conjugué à la troisième personne du pluriel. Une traduction littérale nécessiterait notamment une explication supplémentaire : 'Il faut de la colère pour (...)', 'Tout ce qu'il faut pour (...), c'est votre colère'.

Pour la traduction néerlandaise, nous proposons (tout comme dans la traduction française) de rendre visible «l'Homme» (personnes qui luttent pour les intérêts sociaux des mendiants) qui est le déclencheur indirect de la 
colère : Het enige wat we nodig hebben, is jullie woede - collocation : iemands woede nodig hebben.

Dans l'exemple suivant, le déclenchement de la colère se situe dans la dimension relationnelle 'les autres' - 'Adam'. L'agent externe sont 'les autres' qui veulent la 'ramener à une explosion' chez Adam :

- sprowadzić czyjś gniew do wybuchu czego - ramener la colère de quelqu'un à une explosion de qqc:

- Pozostali z pańskich przyjaciót chcq z wszelka cenę sprowadzić jego gniew do wybuchu geniuszu malarskiego (KW-PL 35)

- Quant aux autres j'ai l'impression qu'ils essaient à tout prix de ramener votre colère à une explosion de génie pictural (KW-FR 294)

En ce qui concerne le degré de l'équivalence de la traduction française de cette collocation, nous constatons une équivalence qui présente une structure analogue mais avec une variation lexicale du Vinch entraînent une signification de 'ranimer', 'provoquer le retour de' (cf. 'ramener') la colère. L'original polonais n'exprimant pas cette nuance, nous proposons la traduction du verbe sprowadzić par conduire ou mener ou amener la colère à une explosion dans laquelle amener adopte le sens d"'entraîner' (PR) (L).

Nous pouvons traduire cette collocation littéralement en néerlandais afin d'exprimer au mieux la propriété relationnelle et l'aspect inchoatif de cette collocation: (iemands) woede tot uitbarsting brengen.

Dans la collocation suivante, nous voyons que l'agent externe (causatif) est à nouveau une personne (cf. Mówca - « l'Homme ») qui demande la colère des mendiant :

- dostarczyć gniewu - donner sa colère

- Dostarczcie tylko gniewu, a znajda się sity, które zdołaja ująć go, wykorzystać i poprowadzić! (KW-PL 75)

- Donnez-nous seulement votre colère, et nous trouverons les forces qui sauront l'utiliser et la guider comme il faut! (KW-FR 341).

Au niveau de la traduction de cette collocation, les équivalences partageant la même aire lexico-sémantique mais avec des structures syntaxiques différentes : dans la traduction française, nous voyons apparaître le pronom possessif votre devant le nom colère. Une traduction littérale, en revanche, est possible mais nécessite également une modification de la structure syntaxique ou l'utilisation d'un partitif: Donnez-nous de la colère.

Nous proposons une traduction néerlandaise littérale par $z$ 'n woede geven de cette collocation ou une traduction qui présente la même signification portée par une structure analogue (Vinch) mais avec une légère variation 
lexicale (ou une équivalence une peu plus lointaine): woede uiten/uiting geven aan je woede ayant la signification de 'exprimer sa colère'.

Dans la version originale (en polonais) de la collocation suivante, l'agent externe qui veut stimuler (wydobyć - extraire) la colère des mendiants, est 'un passant' (przechodzień):

- wydobyć gniew - retrouver la colère

- (...) ja wydobędę (...) caty ten stuszny gniew (KW-PL 70-71)

- Vous leur verrez retrouver (...) leur juste colère (KW-FR 337).

Au niveau de la traduction de cette collocation, nous voyons des équivalences totalement différentes tant du point de vue lexical, syntaxique que sémantique ; dans la version polonaise, un passant (przechodzień) est le sujet de la phrase. C'est lui qui veut 'extraire' cette juste colère des pauvres. Dans la traduction française, nous découvrons les sens des mendiants qui retrouvent leur colère 'perdue'. Tout comme ce fut le cas pour la collocation ramener la colère, nous avons ici également une nuance supplémentaire, qui est celle d'une 'réanimation' de cette émotion. Une traduction littérale s'avère néanmoins possible : 'je ferai sortir cette juste colère'. Cette collocation pourra être traduite en néerlandais par une collocation partageant la même aire lexico-sémantique mais avec des structures syntaxiques différentes (cf. usage de 'er') : de woede eruithalen - Ik haal er die rechtvaardige woede uit.

L'analyse des collocations indiquant la phase initiale de l'expérience de la colère montre que dans la majorité des cas, nous avons à faire à des collocations qui rendent parfaitement le sens/la signification de la collocation, c'est-à-dire la propriété inhérente et relationnelle de la colère (équivalence complète). Nous avons également découvert des exemples où le traducteur remplace la collocation par une collocation plus 'commune' (« connexion plus fixe ») (cf. gniew wzbiera w kimś traduit par se mettre en colère), ou le collocatif par un autre collocatif, ce qui entraîne une (légère) modification du sens par rapport à celui de la collocation dans la langue source. Néanmoins, dans tous ces exemples, une traduction littérale (équivalente) ne compromettrait pas le sens initial de ces collocations.

\subsection{LES COLLOCATIFS EXPRIMANT LA REACTION EMOTIONNELLE DE L'EXPERIENCEUR}

Dans cette partie de notre analyse, nous avons regroupé des collocations avec des verbes collocatifs exprimant la phase de continuation, de déroulement, de développement de l'état émotionnel chez un sujet psychologique. Dans 
le modèle intégré (cf. schéma 3) nous voyons que la 'réaction émotionnelle' sur le 'stimulus' ('causatif'), provoquant la colère, résultera en l'expérience de l'état de la colère/d'une propriété de la colère (aspect duratif/Vcont) qui se situe à un de trois niveaux psychologiques suivants (cf. Schéma 3): a) la cognition (jugement, opinion, connaissance) ; b) la physiologie (symptômes) ; c) le ressenti subjectif.

Comme dans la section précédente, nous avons classifié les collocations avec un collocatif (Vcont) exprimant une de ces trois propriétés.

a. Cognition (jugement, opinion, connaissance) : collocatifs exprimant une attitude cognitive lors de l'expérience de l'émotion :

Dans les collocations suivantes, le verbe chodzić (o) exprime la nuance d'une personne qui porte son jugement sur/perçoit l'état émotionnel ('colère') dans lequel se trouve une autre personne:

- chodzić o (Pański) gniew - s'agir de (votre) colère

- Tak, bo tutaj chodzi o pański gniew (KW-PL 35)

- Il s'agit bel et bien de colère (KW-FR 294)

- A tutaj chodzi tylko o gniew (KW-PL 80)

- Or, c'est de la colère qu'il s'agit ici (KW-FR 346).

Au niveau de la traduction française des collocations nous avons affaire, dans les deux cas, à un collocatif qui exprime l'aspect duratif. Néanmoins, au niveau de la phrase, le traducteur élimine les mots comme pański ('votre'), tutaj ('ici') et tak ('oui') dans la première, et tylko ('uniquement') dans la deuxième.

En néerlandais, nous pouvons rendre le même aspect duratif de cette collocation en utilisant le collocatif : gaan (over)' + N ('woede') : gaan over woede -Ja, want hier gaat et om uw woede / En hier gaat het om woede.

Dans la collocation suivante, le collocatif posiadać exprime l'aspect duratif, exprimant une propriété de la colère qui est constatée chez Adam par « l'Inconnu » (Nieznajomy):

- posiadać gniew - avoir la colère

- Pan posiada ten gniew i to wyczucie. Posiada je Pan z intuicji, z talentu, z geniuszu (KW-PL 36)

- Vous, vous avez cette colère, ce sens de la colère. Grâce à votre intuition, votre talent, votre génie (KW-FR 295).

Au niveau de la traduction française de cette collocation, le traducteur utilise une expression équivalente tant du point de vue lexical, syntaxique que sémantique : posiadać gniew - avoir la colère. 
En néerlandais, l'aspect duratif de cette collocation peut parfaitement être rendu par une traduction littérale (équivalente): U bezit deze woede, dit gevoel.

Une autre possibilité serait de traduire cette collocation par de woede ervaren ('éprouver la colère') qui exprime la perception, le ressentiment de la colère par l'expérienceur qui la possède.

b. Physiologie

Dans toutes les collocations liées à l'expérience physique de la colère, nous avons affaire à une personnification de cette émotion ou l'expression du comportement d'une colère exprimée métaphoriquement. L'émotion devient en quelque sorte un acteur, et révèle un aspect de la manière dont elle est ressentie par le sujet psychologique ou remarquée par un tiers. Dans les exemples polonais cités ci-dessous, les collocatifs (V) expriment l'aspect duratif :

- gniew milczy - taire sa colère/la colère d'autrui

- Dlaczego milczy w was gniew? (KW-PL 74)

- Pourquoi taire votre juste colère? (KW-FR 341).

La traduction française de cette collocation partage la même aire lexicosémantique mais avec des structures syntaxiques différentes: le nom de la 'colère' devient l'objet direct de la phrase. Une traduction littérale 'Pourquoi se tait en vous la colère ?' est effectivement 'moins élégante' du point de vue stylistique. Néanmoins nous pourrions accepter: 'Pourquoi la colère en vous se fait silencieuse?' qui représente le même degré d'équivalence pour des raisons syntaxiques (en français nous avons un verbe pronominal se taire).

Pour la traduction en néerlandais, nous pourrions utiliser l'image de la colère qui, comme la tempête, se calme. Cette nuance est rendue en néerlandais par le prédicat nominal stil worden qui signifie 'devenir silencieux' ce qui donnera: de woede wordt stil. Une traduction littérale par de woede zwijgt est également une traduction possible car elle réfère au silence ou au manque de paroles qui l'accompagnent lorsque la colère est exprimée.

Dans l'exemple suivant, le collocatif (V) exprime l'aspect duratif :

- on [gniew] się czai - il [1'exaspération] se contient

- Na razie ciagle jeszcze czai się on [gniew] [...] (KW-PL 35)

- Pour le moment, il [l'exaspération] se contient encore (KW-FR 294).

En polonais, à l'aide de cette collocation, l'auteur exprime une belle métaphore, représentant la colère comme un animal qui se cache, se tenant prêt à l'attaque. Le traducteur aurait pu se servir d'une traduction littérale: 'Pour le moment, elle est à l'affût' ce qui veut dire 'en train de guetter' (TLFi). 
Néanmoins, il a recours à une traduction avec un degré d'équivalence totalement différent tant du point de vue lexical, syntaxique que sémantique.

En néerlandais, nous pouvons parfaitement rendre la métaphore de la colère qui, comme un animal sauvage, se tient prêt (aspect duratif) avant de 's'accaparer de sa proie': de woede ligt op de loer (= degré d'équivalence complet).

Dans la collocation suivante, le collocatif (V) exprime le déroulement de la colère qui s'étend dans la durée :

- gniew się zerwie - [la colère] devient agissante

- Chodzi o to, aby ostatecznie zerwat się [gniew] (KW-PL 35-36)

- Il faut (...) qu'elle [la colère] devienne agissante (KW-FR 94-95).

Tout comme ce fut le cas pour la traduction de la collocation précédente, nous constatons que le traducteur 'élimine' la métaphore de la colère qui se lève comme le vent (zerwać się) avant la tempête. Ceci peut être associé à l'état physiologique qui accompagne la colère avant qu'elle n'éclate sous forme de tempête (c'est-à-dire en actions ou en paroles). Nous avons donc à faire à un degré d'équivalence qui présente la même signification portée par une structure analogue mais avec une variante lexicale.

En néerlandais, nous pouvons rendre cette propriété (état - aspect duratif) de la colère grâce à une traduction littérale du collocatif et le maintien de l'image de la tempête qui se lève: de woede barst/breekt los.

Dans la collocation suivante, ainsi que dans la traduction française, le collocatif exprime le sens du déroulement de la colère (= aspect duratif/état) qui devrait éclater dans la phase finale:

- gniew wybuchnie - la colère explose

- Przecież wiecie, że gniew musi wybuchna. (KW-PL 102)

- Ignorez-vous que la colère, ça explose? (KW-FR 373).

Dans cet exemple, nous voyons des collocations partageant la même aire lexico-sémantique mais avec des structures syntaxiques différentes : en polonais nous n'avons pas de verbe modal et une phrase d'interrogation comme en français. Néanmoins, le sens est le même, et l'image de la colère qui devrait, en dernière phase de son déroulement, éclater comme une tempête est transmise dans la langue cible.

L'image de cet état/phase peut être préservée par une traduction littérale néerlandaise par: de woede barst uit.

c. Ressenti subjectif:

Dans cette partie nous analysons quelques exemples des collocatifs exprimant les réactions, les comportements personnels de l'individu lors de 
l'expérience de l'émotion. Dans cette catégorie, nous prenons également en compte l'émotion 'colère' comme une émotion avec laquelle l'expérienceur est familiarisé, mais aussi comme un état qui est 'vécu/éprouvé' par d'autres personnes (ici : par les mendiants/les pauvres).

Nous pouvons illustrer cette dimension du 'resenti' par le collocatif suivant exprimant un aspect duratif:

- gniew nurtować kogo - être en proie à l'exaspération

- [...] Rzesze nurtuje wielki, ogromny, bezbrzeżny gniew (KW-FR 35)

- Il [le peuple] est en proie à l'exaspération (KW-PL 295).

La traduction française représente un autre verbe et un autre nom de la colère: exaspération. La signification des deux collocations sont pas tout à fait semblables : en polonais nous avons l'image de la foule rongée par la colère (qualifiée par des adjectifs wielki (grand), ogromny (immense), bezbrzeżny (infini)) qui tourmente, compromet l'équilibre, la cohésion de la foule ( $\mathrm{Pl}$ : rzesze); ceci est rendu par le verbe nurtować ( $\mathrm{Fr}$ : ronger). Par contre en français, la foule (ici traduit par peuple) est absorbée par/est sous l'emprise de ce sentiment violent, traduit par exaspération qui désigne un état de grande irritation, de violente colère. Le collocatif français être en proie de suggère l'image de la colère en tant qu'un rapace. Formulées de façons différentes, les collocations rendent le même sens, celui de personnes qui sont accablées par le sentiment de colère. De ce fait, nous avons à faire à un degré d'équivalence qui présente la même signification portée par une structure analogue mais avec une variante lexicale.

Nous proposons la traduction en néerlandais : in de greep zijn van de woede, ou une autre traduction avec un degré d'équivalence qui rend l'état/ l'aspect duratif exprimé dans la langue source, et qui partage la même aire lexico-sémantique avec des structures syntaxiques différentes ou semblables comme dans l'exemple suivant: overmand worden door woede. En effet, en néerlandais nous devons avoir recours à une construction ou le nom de la colère remplit la fonction du sujet (et non pas d'objet) dans la collocation.

L'analyse des traductions françaises des collocations indiquant la phase $\mathrm{du}$ déroulement/développement de la colère (aspect duratif) montre que dans la majorité des cas, nous avons affaire à des collocations exprimant le même aspect. Le manque d'équivalence complète est principalement dû aux différences syntaxiques entre le polonais et les deux langues cibles. Dans certains cas, le traducteur a recours à un synonyme de colère qui englobe la signification sémantique du verbe lui-même (comme nous avons vu dans l'exemple 
ou le traducteur se sert du mot exaspération pour exprimer l'intensité avec laquelle la colère agit).

\subsection{LES COLLOCATIFS EXPRIMANT LE COMPORTEMENT DE L'EXPÉRIENCEUR}

Dans cette partie de notre analyse, nous avons regroupé des collocations avec des collocatifs exprimant la phase terminale, marquant la fin de l'expérience de la colère.

Tout comme ce fut le cas dans la phase du déclenchement de l'émotion 'colère' - et conforme à la psychologie humaine (= le réel) - une propriété de la colère, exprimée par le colloctif $(\mathrm{V})$ - peut être éprouvée par un 'expérienceur' (une personne ou un groupe), ou causé/suscité par un 'agent externe' (= une personne/des personnes, des évènements).

$\mathrm{Vu}$ que dans la pièce Brat naszego Boga, nous n'avons pas trouvé d'exemples de la colère éprouvée par un 'expérienceur' (une personne ou un groupe) dans cette phase terminale, nous nous limiterons à présenter quelques exemples ou elle est suscitée par un 'agent externe'. Dans cette partie, nous allons également prendre en compte des collocatifs qui ont le sens de la manière dont se sert 1'agent externe'- dans un dernier temps - à savoir: de la colère suscitée chez les mendiants pour atteindre un objectif (social, spirituel ou autre).

\section{Agent externe :}

Dans toutes les collocations suivantes, le collocatif exprime un comportement de 1'“Agent externe' (ici : «l'Iconnu ») qui résulte de l'état de la colère dans lequel trouve un groupe de personnes (ici : les mendiants).

Dans le premier exemple, il s'agit de l'exploitation de la colère par « l'Inconnu » qui veut l'exploiter ou en abuser en vue d'atteindre des objectifs d'ordre social par le biais d'une révolution (ici : des changements sociaux):

- wyzyskać gniew - exploiter la colère

- [...] a co innego wyzyskać ten gniew [...] (KW-PL 80)

- (...) une autre chose est de vouloir l'exploiter (KW-FR 347).

Les collocations présentent un degré d'équivalence complet tant du point de vue lexical, syntaxique que sémantique. Le collocatif exprime le même sens : la colère peut être exploitée.

Cette même propriété (qui sous-tend la signification du collocatif) peut être rendue en néerlandais par une traduction littérale (= niveau d'équivalence complet): de woede uitbuiten. 
Dans le deuxième exemple, l'agent externe est «un passant» (przechodzień) qui veut convaincre son interlocuteur que sa façon de traiter la colère du peuple (= l'éduquer à des fins spirituelles), provoque un affaiblissement de sa colère de sorte qu'il n'aura finalement pas répondu à ses aspirations. Pour cela, l'auteur utilise l'image d'une lame (ostrze) qui transperce la colère, pour ainsi dire. Cette lame peut être associé à la lance qui a transpercé/ fendu le cœur du Christ le Vendredi Saint, déversant les flots de Sa Miséricorde sur l'humanité entière.

- rozszczepić ostrze gniewu - émousser la vigueur de la colère

- [...] rozładowuje, rozszczepia jego [gniew] ostrze (KW-PL 68)

- (...) en émoussant la vigueur (KW-FR 335).

Dans la traduction française, la métaphore avec l'image de la lame a disparu. Le sens de l'affaiblissement de la colère rendu par le verbe émousser qui bien que comprenant la signification 'rendre moins coupant, moins aigu' n'évoque pas immédiatement l'image d'une lame lorsque le mot n'est pas explicitement mentionné. En employant des équivalences totalement différentes tant du point de vue lexical, syntaxique que sémantique et en supprimant la métaphore dans la traduction, nous perdons l'image de la colère fendue par une lame (comme le coeur 'fendu' du Christ) qui aboutit à la transformation de la colère (du peuple) en miséricorde, que le Frère Albert, à la suite du Christ, voyait comme le seul moyen de vaincre l'iniquité dans le monde.

En ce qui concerne la traduction néerlandaise, nous proposons une traduction équivalente afin de préserver l'image de la métaphore dans le texte original : de woede met een lans doorboren/doorsteken ('percer/fendre la colère avec une lance').

Dans la collocation suivante il s'agit de la même personne que dans l'exemple précédent qui - en tant qu'Agent extérieur - cherche à abuser de l'état de la colère que se manifeste chez les mendiants. Nous avons donc affaire à une nouvelle relation de la colère dont on peut abuser :

- nadużyć gniewu - exploiter la colère

- [...] naduzyć go (KW-PL 80)

- (...) une autre est de vouloir l'exploiter en l'utilisant comme un simple outil (KW-FR 347).

La traduction française présente une structure analogue mais avec une variation lexicale: l'équivalent français exact pour traduire naduzyć est abuser mais le traducteur a choisi exploiter.

Abuser signifie 'user mal ou avec excès' (L). Exploiter signifie 'utiliser une situation, un événement à son avantage' (L). Uniquement dans le cas ou 
l'objet est une personne, le verbe exploiter est synonyme d'abuser: 'se servir de quelqu'un d'une manière abusive' (L). Au cas où l'objet de la phrase est une chose, exploiter a une sens positif: profiter de, faire valoir (une chose), tirer parti de (PR). Vu que nous avons affaire à une chose (cf. 'la colère'), nous opterions dans ce cas pour le verbe abuser.

Nous proposons une traduction néerlandaise avec un degré d'équivalence complet qui rendra le mieux la propriété de la colère dans cette phase : de woede misbruiken.

Dans les exemples ci-dessous, le collocatif wychować exprime un comportement de 1' 'agent externe' (dans ce cas : le Frère Albert) qui résulte comme dans l'exemple précédent - de l'état de la colère des mendiants. Ce dernier veut - contrairement à " l'Inconnu ", éduquer la colère pour des objectifs supérieurs à ceux de «l'Inconnu » : la sortie de la misère grâce à l'aide de Dieu.

- wychować gniew / éduquer la colère

- Świadomie ja chce jedynie wychować ten gniew (KW-PL 347)

- Co innego przecież znaczy wychować stuszny gniew (KW-PL 347)

- Tak, tak, będzie go pan wychowywat po to, ażeby znów sttumić cała jego moc (KW-PL $80-81)$

- Ce que je cherche sciemment à faire, c'est éduquer la colère (KW-FR 347)

- Car autre chose est de vouloir éduquer la colère (...) (KW-FR 347)

- Bien sûr, vous l'éduquerez comme d'habitude: pour étouffer sa force (KW-FR 347).

Dans la traduction française, les trois collocations avec le collocatif wychować représentent un degré d'équivalence sémantique et lexicale complète, ayant été traduit par le verbe éduquer. Au niveau de la traduction de la phrase, l'adjectif stuszny (juste) n'a pas été traduit dans le deuxième exemple. Au niveau de l'équivalence de la syntaxe, nous constatons uniquement une adaptation dans la traduction française du premier exemple. Les deux autres représentent également un degré complet d'équivalence syntaxique.

Afin de rendre le même sens de la propriété de la 'colère' que l'on peut 'éduquer' (sens positif) nous proposons une traduction néerlandaise avec un degré d'équivalence complet (lexical, syntaxique et sémantique): de woede opvoeden.

Une autre solution serait de traduire cette collocation par de woede in goede banen leiden qui a plutôt le sens de 'canaliser', 'orienter', 'diriger' mais qui pourrait être plus approprié lorsque nous parlons de sentiments. 


\section{CONCLUSION}

Cette étude comparative des collocations verbo-nominales $(\mathrm{V}+\mathrm{N} / \mathrm{N}+\mathrm{V})$ avec le $\mathrm{N}$ de base désignant l'émotion de colère - qui dans toutes les langues renvoie à la même réalité extralinguistique (cadre référentiel) - a démontré le lien entre le verbe collocatif $(\mathrm{V})$ et le mot de base $(\mathrm{N})$ et nous rend capable de 'détecter' des propriétés spécifiques de cette émotion qui peuvent être éprouvées [de manière individuelle (par une ou plusieurs personnes, un groupe de personnes)] et vécues (suscitées, ressenties et éteintes ou exploitées/manipulées) dans un contexte relationnel.

Comme nous l'avons noté dans l'analyse des exemples de traductions des collocations verbo-nominales avec le nom désignant la colère, le traducteur pourra avoir recours à un équivalent de la collocation verbo-nominale (= collocation la plus courante qui se trouve dans un dictionnaire) ancré dans la langue cible et bien connu du lecteur. Tout au long de cette analyse nous avons constaté que le traducteur a recours à cette stratégie dans le cas des collocations liées à la phase initiale de l'expérience de la colère où il remplace la collocation par une collocation plus 'commune' ('connexion plus fixe') dans la langue cible.

Dans le cas où il n'existe pas d'équivalent, les résultats de cette analyse nous montrent qu'il peut faire appel au cadre conceptuel de l'émotion (ou d'un autre concept) afin de trouver un équivalent analogue et «linguistiquement et stylistiquement acceptable " dans la langue cible. En adoptant cette deuxième solution/stratégie, il obtiendra des traductions plus proches du sens des collocations en langue source, ce qui est particulièrement le cas de collocations liées à l'expérience physique de la colère - où nous avons affaire à une personnification de cette émotion - et celles liées à la phase marquant la fin de l'expérience de la colère.

Le fait que de nombreuses collocations n'ont pas d'équivalent dans la langue cible témoigne en un certain sens de l'immense créativité de l'auteur dans la manière dont il réussit à personnifier l'émotion 'colère' dans ses différentes phases et dimensions (cf. personnel et relationnel). Néanmoins, le manque de courage/d'audace de la part du traducteur de traduire certaines métaphores littéralement compromet la richesse des images évoquées par l'écrivain dans le texte source.

$\mathrm{Au}$ terme de cette analyse, nous pouvons cependant affirmer qu'une approche sémantico-discursive dans l'analyse comparative de la traduction de certaines unités lexicales - telles les collocations - pourrait être adoptée comme 
point de départ pour définir les critères permettant d'évaluer la qualité d'une traduction. Il faut espérer que cela stimulera la recherche dans cette direction.

\section{BIBLIOGRAPHIE}

Cabré, M. Teresa. « On diversity and terminology ». Terminology vol. 2, nº 1, 1995, pp. 1-17.

Coppin, Géraldine, David Sander. «Théories et concepts contemporains en psychologie de l'émotion ». Systèmes d'interaction émotionnelle, ed. Catherine Pelachaud. Hermès Science publications-Lavoisier, 2010, pp. 25-56. https://archive-ouverte.unige.ch/unige:34368. Accès 1.10. 2021.

Cruse, D. Alan. Lexical semantics. Cambridge University Press, 1986.

Ekman, Paul. « An argument for basic emotions ». Cognition \& Emotion, nº 6, 1992, pp. 169-200.

Geerts, Guido \& den Boon, Ton. van Dale Groot Woordenboek der Nederlandse Taal - Dertiende herziene uitgave, Van Dale Lexicografie, 1999.

González Rey, Isabel. « Le 'double' principe d'idiomacité en traduction littéraire ». Revista de Filología, $\mathrm{n}^{\mathrm{0}} 32$, 2014, pp. 227-244.

Hausmann, Franz J. « Le dictionnaire de collocations ». Wörterbücher: ein internationales Handbuch zur Lexicographie. Dictionaries. Dictionnaires, eds. Franz J. Hausmann, Oskar Reichmann, Herbert E. Wiegand and Ladislav Zgusta, De Gruyter, 1989, pp. 1010-1019.

James, William. «What is an emotion? ». Mind, $\mathrm{n}^{\circ}$ 9, 1884, pp. 188-205. psychclassics.yorku.ca/ James/emotion.htm. Accès 1.02.2021.

Krzyżanowska, Anna \& Balaţchi, Raluca-Nicoleta. «Traduire les émotions. Introduction ». Lublin Studies in Modern Languages and Literature, $\mathrm{n}^{\circ}$ 44(1), 2020, pp. 1-9.

Krzyżanowska, Anna. "Sur la place des noms d'émotion dans les études contrastives ». Studia Romanica Posnaniensia, vol. 42, n 5, 2015, pp. 67-79.

Lerat, Pierre. Les langues spécialisées, Presses Universitaires de France, 1995.

Maryniarczyk, Andrzej. « Rola języka naturalnego w metafizyce realistycznej ». Gaudium in litteris, red. Stanisław Janeczek, Wanda Bajor, Michał Maciołek, Wydawnictwo KUL, 2009, pp. 665-677.

Maryniarczyk, Andrzej. Metafizyka a ontologie. Wydawnictwo KUL, 2015.

Matsumoto, David, \& Ekman, Paul. « Basic emotions ». The Oxford companion to emotion and the affective sciences, eds. David Sander, Klaus R. Scherer, Oxford University Press, 2009, pp. 69-73.

Mel'čuk Igor, Clas André, Polguère Alain. Introduction à la lexicologie explicative et combinatoire. Duculot, 1995.

Nussbaum, Martha C. «Emotions as judgements of value and importance ». What is an emotion?, ed. Robert C. Solomon, Oxford University Press, 2003.

Olszewska, Maria. « Piękno Miłosierdzia. Dramaturgiczne wizerunki Brata Alberta ». Zeszyty Naukowe KUL, vol. 61, n 2, 2018, pp. 121-143.

Scherer, Klaus R. «What are emotions? And how can they be measured? ». Social Science Information, vol. 44, $\mathrm{n}^{\circ}$ 4, 2005, pp. 695-729.

Solomon, Robert C. «Emotions and choice ». Review of metaphysics, vol. 28, n 1, 1973, pp. 20-41.

Stoessel, Catherine. L'émotion esthétique dans la relation art-thérapeutique au sein d'un atelier d'artsplastiques dans un centre pénitentiaire de très haute sécurité. Psychologie et comportements. Université Sorbonne Paris Cité, 2017. 
Śliwa, D. «Une approche cognitive des collocations ». Translation and Meaning, Part 6. Proceedings of the Lodz Session of the 3rd International Maastricht-Lodz Duo Colloquium on "Translation and Meaning”, Held in Lodz (Poland), 22-24 September 2000, eds. Marcel Thelen, Barbara Lewandowska-Tomaszczyk, Hogeschool Zuyd Maastricht School of translation and Interpreting, 2002, pp. 291-296. www.hszuyd.msti.translation-and-meaning.nedweb.com/. Accès 17.02.2021.

Śliwa, Dorota. " Coeur et sentiments: une étude cognitive des collocations françaises et polonaises ». Parler des emotions : entre langue et litterature, dir. Anna Krzyżanowska, Renata Jakubczuk, Wydawnictwo UMCS, 2011, pp. 191-199.

Śliwa, Dorota. «Testament : entité - concept - termes français et polonais dans leurs relations pluridimensionnelles ». Roczniki Humanistyczne, vol. 61, c. 8 , 2013, pp. 131-147.

Śliwa, Dorota. « Parlons cerises : les prédicats et les relations dans les définitions lexicographiques et dans les énoncés définitoires ». Białostockie Archiwum Językowe, vol. 15, 2015, pp. 413-427.

Tutin Agnès \& Grossmann Francis. "Collocations régulières et irrégulières : esquisse de typologie du phénomène collocatif ». Revue Française de Linguistique Appliquée, vol. 7, nº 1, 2002, pp. 7-25.

\section{TEXTES SOURCES}

KW- PL : Wojtyła Karol. Brat naszego Boga. Wydawnictwo Św. Stanisława BM, 1989.

KW-FR : Wojtyła Karol. Poèmes, Théâtre: La Boutique de l'Orfèvre-Frère de Notre Dieu, Écrits sur le théatre, Avant-Propos de Jean Offredo - avant-propos et trad. de Zofia Bobowicz et Jean Offredo, Cana/Cerf, 1998.

\section{DICTIONNAIRES GÉNÉRAUX}

Van Dale Groot Woordenboek van de Nederlandse taal, eds. Ton den Boon, Ruud Hendrickx \& Nicoline van der Sijs, Van Dale Uitgevers. 3 delen, 15e druk, 2015.

(L), dictionnaire en ligne : https://www.larousse.fr/encyclopedie/divers/\%C3\%A9lectronique/46278. Consulté en 01.02.2021.

(PR) Le Petit Robert. Dictionnaire alphabétique et analogique de la langue française, red. A. Rey et J Rey-Debove. Dictionnaires Le Robert. 1986.

(TLFi) Le Trésor de la langue française informatisé, version électronique du Trésor de la langue française, dictionnaire de référence des XIXe et XXe siècles en 16 volumes, réalisée par le laboratoire ATILF (Analyse et traitement informatique de la langue française), [En ligne], achevée en 2004, http://atilf.atilf.fr/tlf.htm. Consulté en 01.02.2021.

\section{COLLOCATIONS VERBO-NOMINALES AVEC GNIEW ('COLÈRE') DANS BRAT NASZEGO BOGA (KAROL WOJTYLA) ET LEUR TRADUCTION EN FRANÇAIS ET EN NÉERLANDAIS}

$$
\text { Rés u mé }
$$

Cet article rend compte d'une analyse de la traduction de collocations avec le nom gniew ('colère') dans la pièce de théâtre de Karol Wojtyła Brat naszego Boga (Frère de notre Dieu) en français, et propose des traductions de ces collocations en néerlandais. 
Pour l'analyse sémantico-discursive des collocations avec gniew ('colère'), nous avons établi un modèle méthodologique dans lequel nous avons intégré - à partir de la définition de la colère par Eckman - des éléments de cette émotion (en tant que processus) et leur réalisation linguistique qui se présente sous la forme d'une collocation verbo-nominale (du type $\mathrm{V}+\mathrm{N}$ ou $\mathrm{N}+\mathrm{V}$ ) selon l'approche cognitive de la collocation proposée par Śliwa.

L'analyse des collocations ouvre une perspective contrastive afin de repérer d'éventuelles difficultés dans la traduction des collocations verbo-nominales avec gniew. Pour faciliter une description plus détaillée du degré de correspondance de la traduction de ces collocations, nous avons eu recours au schéma de Camugli Gallardo.

Cette étude nous permet d'affirmer qu'une approche sémantico-discursive appliquée à l'analyse comparative de la traduction de certaines unités lexicales; telles les collocations - pourrait être adoptée comme point de départ pour définir les critères permettant d'évaluer la qualité d'une traduction.

Mots clé : noms de l'émotion; colère ; structure ontologique ; collocations verbo-nominales ; stratégies de traduction; équivalence.

\author{
KOLOKACJE CZASOWNIKOWE Z RZECZOWNIKIEM GNIEW \\ W SZTUCE BRAT NASZEGO BOGA KAROLA WOJTYŁY \\ ORAZ ICH TŁUMACZENIA NA JĘZYK FRANCUSKI I NIDERLANDZKI
}

$$
\text { Streszczenie }
$$

Niniejszy artykuł przedstawia analizę tłumaczenia kolokacji z rzeczownikiem gniew w dramacie Karola Wojtyły Brat naszego Boga na język francuski oraz propozycje przekładów tych kolokacji na język niderlandzki.

Do analizy semantyczno-dyskursywnej kolokacji z rzeczownikiem gniew przyjęto model metodologiczny, w którym zintegrowano - w oparciu na definicji gniewu Ekmana - elementy tej emocji (jako proces) oraz ich językową realizację w postaci kolokacji czasownikowo-rzeczownikowej (typu $\mathrm{V}+\mathrm{N}$ lub $\mathrm{N}+\mathrm{V}$ ) zgodnie z kognitywnym podejściem do kolokacji zaproponowanym przez Śliwę.

Studium otwiera perspektywę kontrastywną w celu zidentyfikowania ewentualnych trudności w tłumaczeniu kolokacji czasownikowo-rzeczownikowych z rzeczownikiem gniew. Aby ułatwić bardziej szczegółowy opis stopnia 'ekwiwalencji' w przekładzie tych kolokacji, odwołaliśmy się do schematu Camugli Gallardo.

Analiza ta pozwala nam stwierdzić, że podejście semantyczno-dyskursywne zastosowane do analizy porównawczej przekładu pewnych jednostek leksykalnych, takich jak kolokacje - może zostać przyjęte jako punkt wyjścia do określenia kryteriów oceny jakości przekładu.

Słowa kluczowe: nazwy uczuć; gniew; struktura ontologiczna; kolokacje czasownikowo-rzeczownikowe; ekwiwalencja; strategie thumaczeniowe. 